\title{
HARDWARE AND SOFTWARE PC SKILLS APPLIED TO A SOUND CANCELLATION PROJECT
}

\author{
Sean Daly and Roman Stemprok \\ University of North Texas
}

\begin{abstract}
Developed societies experience an increased level of noise pollution. A Sound Cancellation project was incorporated into a 2 hour-credit Electronics Specials Problems course. The active control of sound is a solution by making an anti-noise for every known noise volume. The antinoise signal will cancel itself along with the noise. A computer runs an adaptive noise control algorithm in assembly code. The program takes input and determines the corresponding value for a cancellation sound output of analog to digital and digital to analog conversions with sine wave and sound 8-bit I/O.

Introduction

Sound Cancellation is not a new concept in combating noise pollution. Many devices use sound cancellation, but are limited in scope, individual pair of sound cancellation device to each listener. Active Noise Cancellation (ANC) is not a new concept; different consumer products and projects have incorporated noise cancellation. The most successful environments for ANC have been for enclosed spaces such as ducts, vehicle cabins, exhaust pipes, and headphones ${ }^{9}$. However, most of these products have not yet made the transition into successful commercial products. ANC headphones use destructive interference to cancel low-frequency noise while still allowing the user to hear mid- and high-frequency sounds such as conversations and warning sirens. The headphone system comprises a pair of earmuffs, containing speakers, and one or more small circuit boards. Some headphones include a built-in battery pack, and many allow exterior signal inputs such as music or voice communications 9 . Another application is active control mufflers for industrial engine exhaust stacks. Active control mufflers have seen years of service on commercial compressors and generators. As unit prices for active automobile mufflers have fallen, several automobile manufacturers are considering active mufflers for future production cars ${ }^{6}$. Large industrial fans have benefited from active control. Speakers placed around the fan intake or outlet not only reduce low-frequency noise downstream and/or upstream, but they also improve efficiency to such an extent that they pay for themselves within two years. The idea of canceling low-frequency noise inside vehicle cabins has received much attention. Most major aircraft manufacturers are developing such systems, especially for noisy propeller-driven aircraft ${ }^{9}$. Speakers in the wall panels can reduce noise generated as the propeller tips pass by the aircraft fuselage. Systems by Noise Cancellation Technologies now comes as standard equipment on the new Saab 2000 and 340B + aircraft. The key advantage is the savings in weight, active treatments
\end{abstract}


using electronic components compared to passive treatments that require heavier materials that reduce noise.

Automobile manufacturers are considering active control for reducing low-frequency noise in car interiors. Car stereo speakers superimpose cancellation signals over the normal music signal to cancel muffler noise and other sounds. Lotus produces a system for sale to other automobile manufacturers. Unit cost is a major consideration for automobile use. While such systems are not at all common, at least one vehicle (currently offered only in Japan) includes such a system as a factory option ${ }^{9}$. Sound cancellation in a large room would be much more efficient then each individual having a device. There are sound cancellation devices that can cancel a point or area in a room, but not any that can cancel a whole room. The issue with canceling the outside sound for a whole room is the mix complexity of different sounds different areas from outside the room. The solution is to microphone points around the room and sound cancel at those points, thus lowering the overall sound noise coming into the room. The key is how to sync the input microphone to the output speaker. The wave shape is changed as a sound wave passes through a wall. Factors that determine how much the wave is changed is based on the type of material used for the walls. Using a computer interfaced with the input and output, an algorithm can compensate for the wave change and effectively cancel it. This computer interfacing should allow for flexibility between the input and output distance. Allowing for the possibility choosing your cancellation points for each room environment unique.

\begin{tabular}{|l|c|c|c|}
\hline \multicolumn{1}{|c|}{ Source } & Intensity & Intensity Level & $\begin{array}{c}\text { \# Times Greater } \\
\text { Than TOH }\end{array}$ \\
\hline Threshold of Hearing (TOH) & $1 * 10^{-12} \mathrm{~W} / \mathrm{m}^{2}$ & $0 \mathrm{~dB}$ & $10^{0}$ \\
\hline Rustling Leaves & $1^{*} 10^{-11} \mathrm{~W} / \mathrm{m}^{2}$ & $10 \mathrm{~dB}$ & $10^{1}$ \\
\hline Whisper & $1 * 10^{-10} \mathrm{~W} / \mathrm{m}^{2}$ & $20 \mathrm{~dB}$ & $10^{2}$ \\
\hline Normal Conversation & $1 * 10^{-6} \mathrm{~W} / \mathrm{m}^{2}$ & $60 \mathrm{~dB}$ & $10^{6}$ \\
\hline Busy Street Traffic & $1 * 10^{-5} \mathrm{~W} / \mathrm{m}^{2}$ & $70 \mathrm{~dB}$ & $10^{8}$ \\
\hline Vacuum Cleaner & $1 * 10^{-4} \mathrm{~W} / \mathrm{m}^{2}$ & $80 \mathrm{~dB}$ & $10^{9.8}$ \\
\hline Large Orchestra & $6.3^{*} 10^{-3} \mathrm{~W} / \mathrm{m}^{2}$ & $98 \mathrm{~dB}$ & $10^{10}$ \\
\hline Walkman at Maximum Level & $1 * 10^{-2} \mathrm{~W} / \mathrm{m}^{2}$ & $100 \mathrm{~dB}$ & $10^{11}$ \\
\hline Front Rows of Rock Concert & $1 * 10^{-1} \mathrm{~W} / \mathrm{m}^{2}$ & $110 \mathrm{~dB}$ & $10^{13}$ \\
\hline Threshold of Pain & $1 * 10^{1} \mathrm{~W} / \mathrm{m}^{2}$ & $130 \mathrm{~dB}$ & $10^{14}$ \\
\hline Military Jet Takeoff & $1 * 10^{2} \mathrm{~W} / \mathrm{m}^{2}$ & $140 \mathrm{~dB}$ & $10^{16}$ \\
\hline Instant Perforation of Eardrum & $1 * 10^{4} \mathrm{~W} / \mathrm{m}^{2}$ & $160 \mathrm{~dB}$ & \\
\hline
\end{tabular}

Table I: Sound Level Examples ${ }^{8}$

Sound is a real world element that is the result of molecules moving through a medium in ordered motion. This motion moves in the form of a wave, and has inherited properties defined by physics. Sound moves from its source to a receiver as a "wave", which can be defined as "a disturbance propagating through a medium." The medium can be almost any matter that vibrates. A disturbance means that the vibrating object changes the motion of the particles (atoms or molecules) of the medium. Propagation means the wave moves from one place to another. A disturbance or noise can be measured by its intensity, displacement and pressure. Intensity measures the energy transported over an area $I=\frac{\text { Energy }}{\text { Time }} *$ Area , or $I=\frac{\text { Power }}{\text { Area }}$ expressed in units of Watts/ meter ${ }^{2}$. The sound intensity from a point source of sound will obey the inverse square law if there are no reflections or reverberations. That means the area (A) in the equation 
$(\mathrm{I}=\mathrm{P} / \mathrm{A})$ becomes the surface area of a sphere. The sound is emitted uniformly in all directions. A decibel scale measures intensity (which is the measured relationship between two values of intensity or power).

Wave interference happens when two waves travel in the same medium. The two waves combine in the medium and result in one wave product. When two waves crest at the same point the combined effect is that the crest will be the sum of the two waves. When two waves trough at the same time they add together to form a deeper trough. These two wave events are forms of constructive interference. In the case where a trough meets a crest at the same point the effect is called destructive interference. These two waves take power away from each other and effectively cancel each other to zero if exactly in synch. This experiment uses this phenomenon to cancel noise.

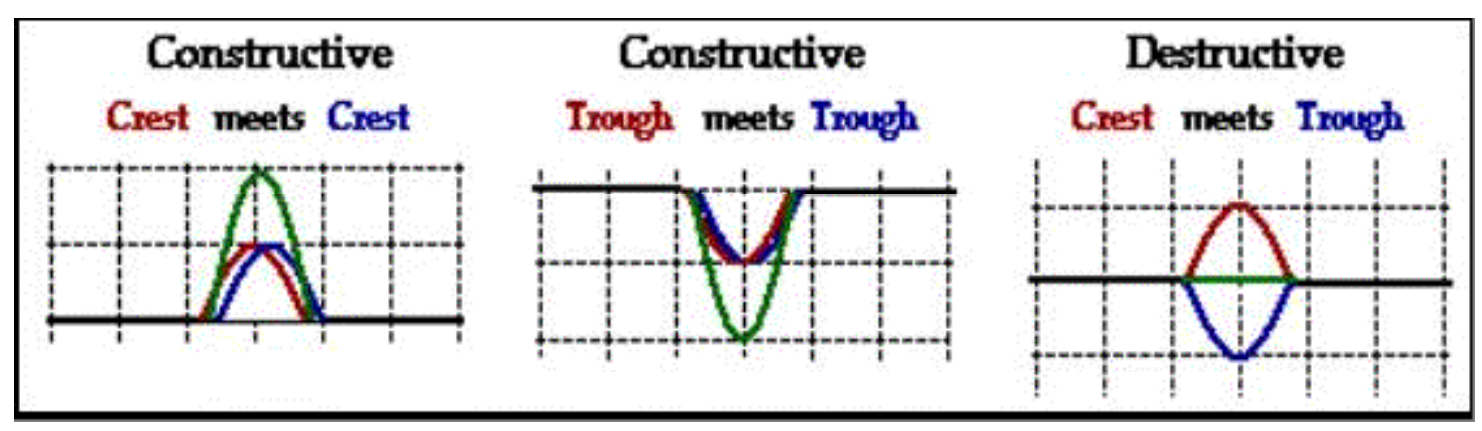

Figure 1: The Concept of Constructive and Destructive Interference ${ }^{2}$

\section{Experimental Setup}

Background on Hardware Components and Software Design:

The computer used is a $180 \mathrm{~Hz}$ Pentium-S processor, with a motherboard ISA slot used for the interfacing card. The card is called a PC Bus Extender Card (I/O 300-31F) ${ }^{1}$. The PC Bus Extender Card is used to take a signal from the PC and sends it via cable to a PC Interface Trainer Module.

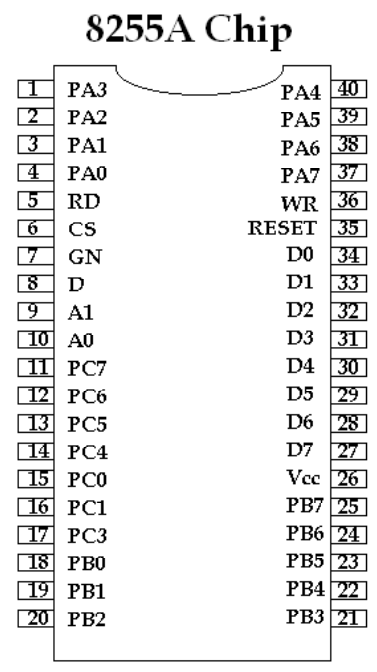

Figure 2: 8255 Chip Layout 
The card only allows access to port addresses 300-31Fh, and can be used with any IBM or otherwise compatible PC with ISA slots. The PC INTERFACE TRAINER MODULE contains onboard 8255 and $8253 / 4$ chips.

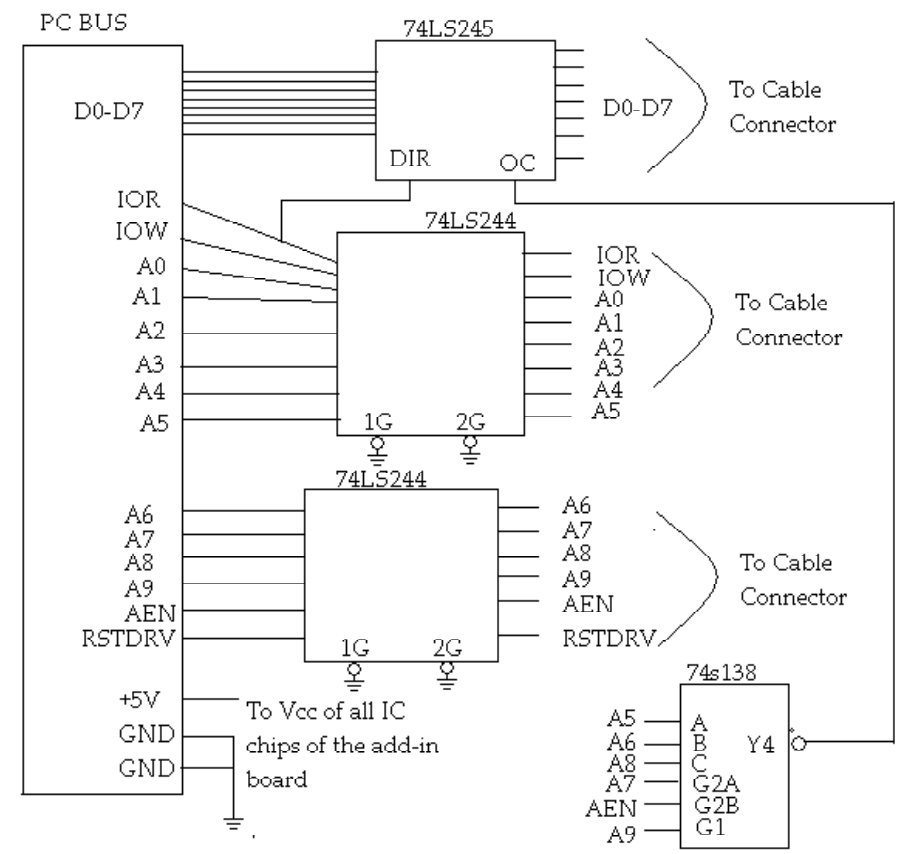

Figure 3: PC Bus Extender Diagram

The 8255 chip is a 40-pin DIP integrated circuit which has three 8-bit ports that can separately receive input and output (the third port being split into high and low to enable input and output at the same time). The 8255 can also be used to interface the CPU to devices for handshaking signals. The software component used in programming the computer is an assembly program. The goal was to have the least number of process lines to increase the programs process speed to meet the speed of faster sound frequencies.

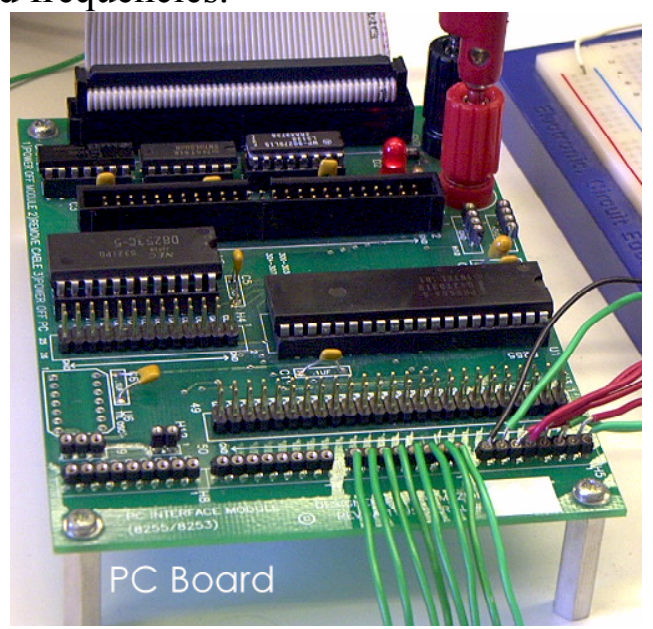

Figure 4: Actual PC Board

Important parts of the program include the Trainer's port initialization, input values (from the 
input circuit), mathematical calculation, and the value back output to the Trainer (and on to the output circuit).

\begin{tabular}{|c|c|c|c|}
\hline portA & $d w$ & $300 \mathrm{~h}$ & \\
\hline portB & $d w$ & $301 \mathrm{~h}$ & \\
\hline control & $d w$ & $303 \mathrm{~h}$ & \\
\hline control-value & $\mathrm{db}$ & $90 \mathrm{~h}$ & \\
\hline pitch_in & $\mathrm{db}$ & & \\
\hline pitch_out & & $\mathrm{db}$ & ? \\
\hline ze & $\mathrm{db}$ & OFeh & \\
\hline
\end{tabular}

Ports A and B and the Control Register are located at memory locations 300h, 301h, and 303h. The control value is set as $10010000 \mathrm{~b}(90 \mathrm{~h})$. This control value sets the ports to their $\mathrm{I} / \mathrm{O}$ function. D7=1; puts the Trainer into Input / Output Mode. D6-5=00; sets the Trainer in basic $\mathrm{I} / \mathrm{O}$ mode (other options would be $\mathrm{I} / \mathrm{O}$ with handshaking and outputting data with handshaking). D4=1; sets all 8-bits of Port A to Input mode. D3, D2, D0 all relate to Port C which is not being used, so all three are set to 0 . D1=0; sets Port B to Output mode. PITCH_IN and PITCH_OUT were included to keep the input and output values separate to keep data integrity in the PC registers. The variable ZERO_PITCH is the value that will be subtracted to determine the inverse value. The value is FEh. The half value, $0 \mathrm{Fh}$, will be the zero point to correspond with the zero point on the ADC, which is the value $0 F$.

\begin{tabular}{|llll|}
\hline$\ldots$ & cmp & al,7fh & ;7F is value for zero point \\
je & step3 & ;jump if equal to zero-pitch \\
& cmp & al,Offh & ;if equal to FF value \\
je & step2a & ;jumps to manual change to zero \\
& mov & bl,zero_pitch & ;place zero-pitch in 'bl' reg \\
& sub & bl,al & ;find difference from zero-pitch \\
& mov & pitch_out,bl & ;save pitch out \\
\hline$\ldots$ & & & \\
\hline
\end{tabular}

As the program becomes more complex, it will be necessary to use faster processor speeds to keep up with the higher sound frequencies. The inverting program must finish receiving, computing, and outputting data in no less than $.5 \mathrm{~ms}$ (which equates to $2 \mathrm{KHz}$ ) to be effective. This section of code is the inverting process. The first comparison checks for the zero point. If the value already equals the zero point it will return the same value to the output. The second comparison checks for the value FFh. This is the only value that will not correctly correspond to its inverse value using this method. If this value is found, the program will jump to a step that will set these values to $00 \mathrm{~h}$ to fit with the inverted input value FEh (which is $00 \mathrm{~h}$ ). Calculation for the rest of the values are subtracted from the zero pitch (FEh) and the result saved in the variable OUT_PITCH. The program sends the OUT_PITCH value to Port B where it is sent to the Output Interfacing Circuit. This program is basic in design and is flexible to allow for changing the environment. A delay can be added to the beginning of the program to account for 
microphone to speaker distance. Variables PITCH_IN, PITCH_OUT and ZERO_PITCH can be manipulated to account for sound entering or traveling through walls based on how the wall's material affects the wave.

Input and Output Interface Circuit Design: Input and output interfacing circuits will convert the analog input signal into a digital signal that the PC can invert, and back from digital to analog to the speaker. Each side of the interface was centered on TTL chips that convert Analog / Digital signals. The circuit design was to take advantage of the $5 \mathrm{~V},-5 \mathrm{~V}$ source provided by the computer. In this experiment an external voltage source was used to speed-up design time. The Input Circuit consists of a 1.5V-powered microphone, $741 \mathrm{Op}$-Amp, Analog to Digital Converter 0804ADC, and the necessary resistors and capacitors. The output from the microphone is between $-100 \mathrm{mV}$ to $+100 \mathrm{mV}$, and amplified by a factor of 470 through the Op-Amp to the $\mathrm{ADC}$. The ADC configuration was set for an input range of $+/-5 \mathrm{~V}$, with a conversion factor of $667 \mathrm{~Hz}$ (1/RC per the Datasheet). The 8-bit ADC output is connected to Port A of the PC BOARD.
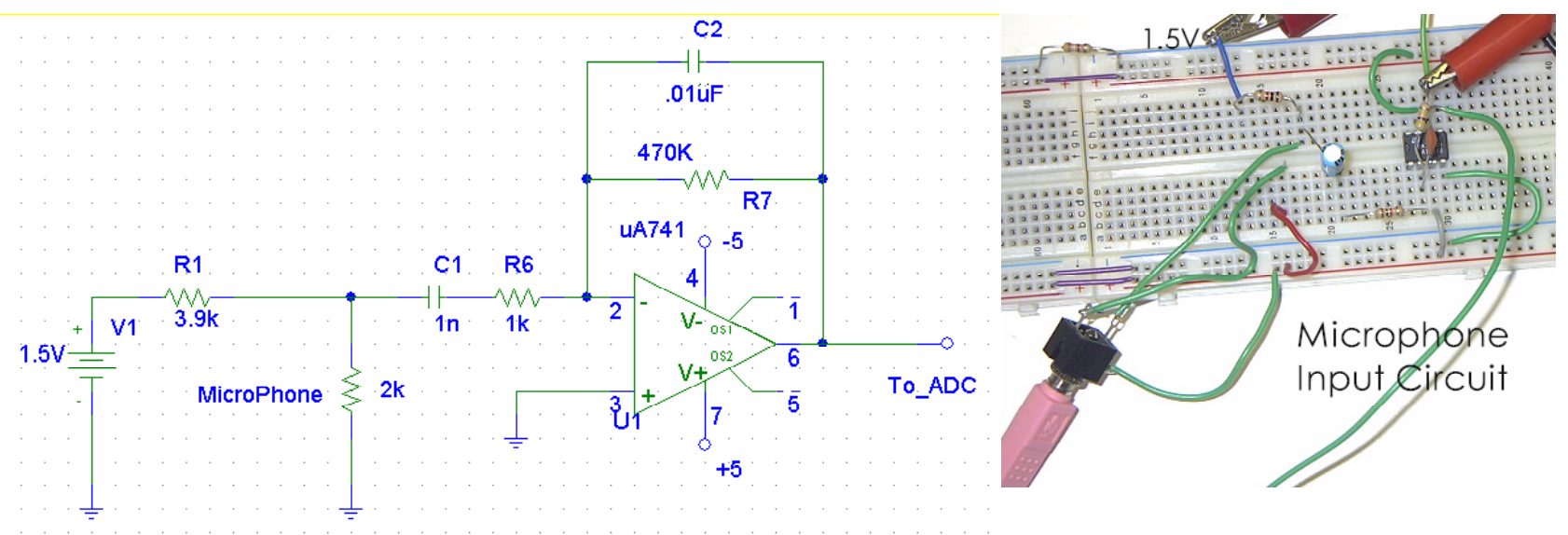

Figure 5: Microphone Input Circuit
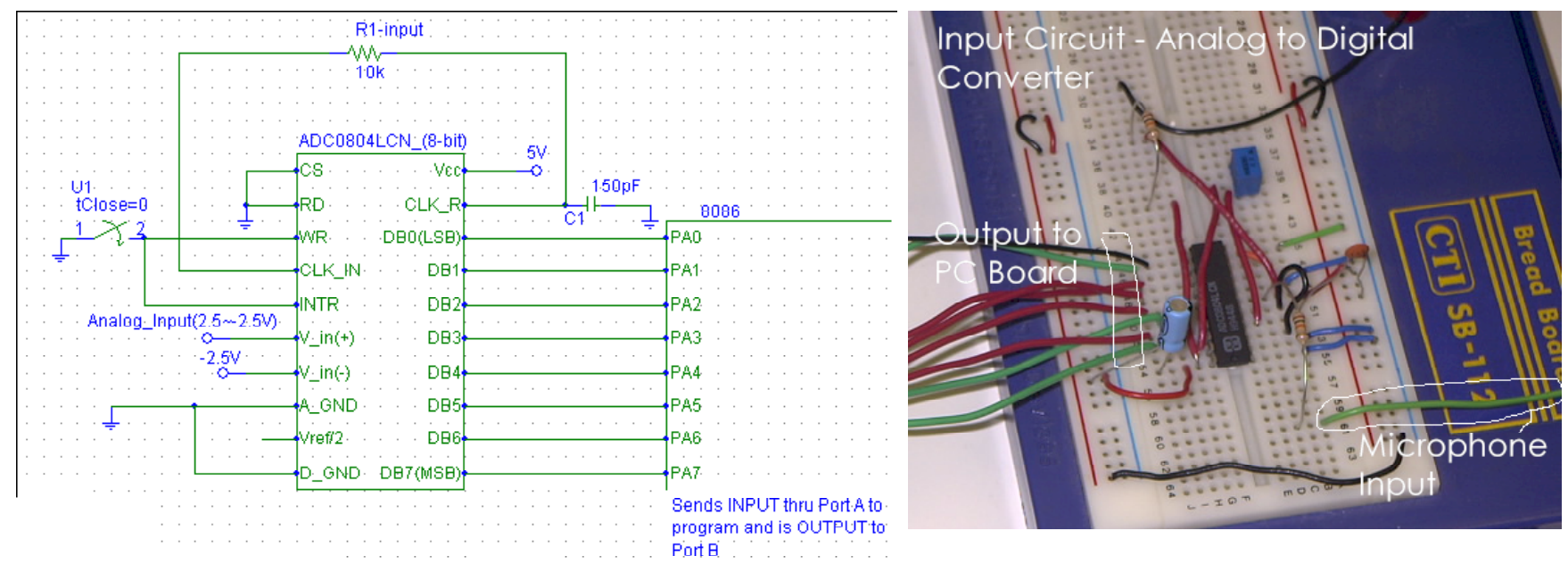

Figure 6: Input Circuit to A/D Converter

The Output circuit consists of a 557 Digital to Analog Converter (DAC), (2) 741 Op-Amps, self

Proceedings of the 2003 American Society for Engineering Education Annual Conference \& Exposition Copyright (C) 2003, American Society for Engineering Education 
powered speakers and the necessary resistors and capacitors. The 8-bit signal from Port B on the PC BOARD is converted through the 557DAC. The $0-2.55$ volt analog signal from the DAC is run through two inverting Op-Amps. The first Inverting Op-Amp subtracts 1.25 volts from the signal, which sets the voltage range to $+/-1.25$ volts. The second Inverting Op-Amp has a gain of -2 , and sends a signal of $+/-2.55 \mathrm{~V}$ to the self-power speakers.
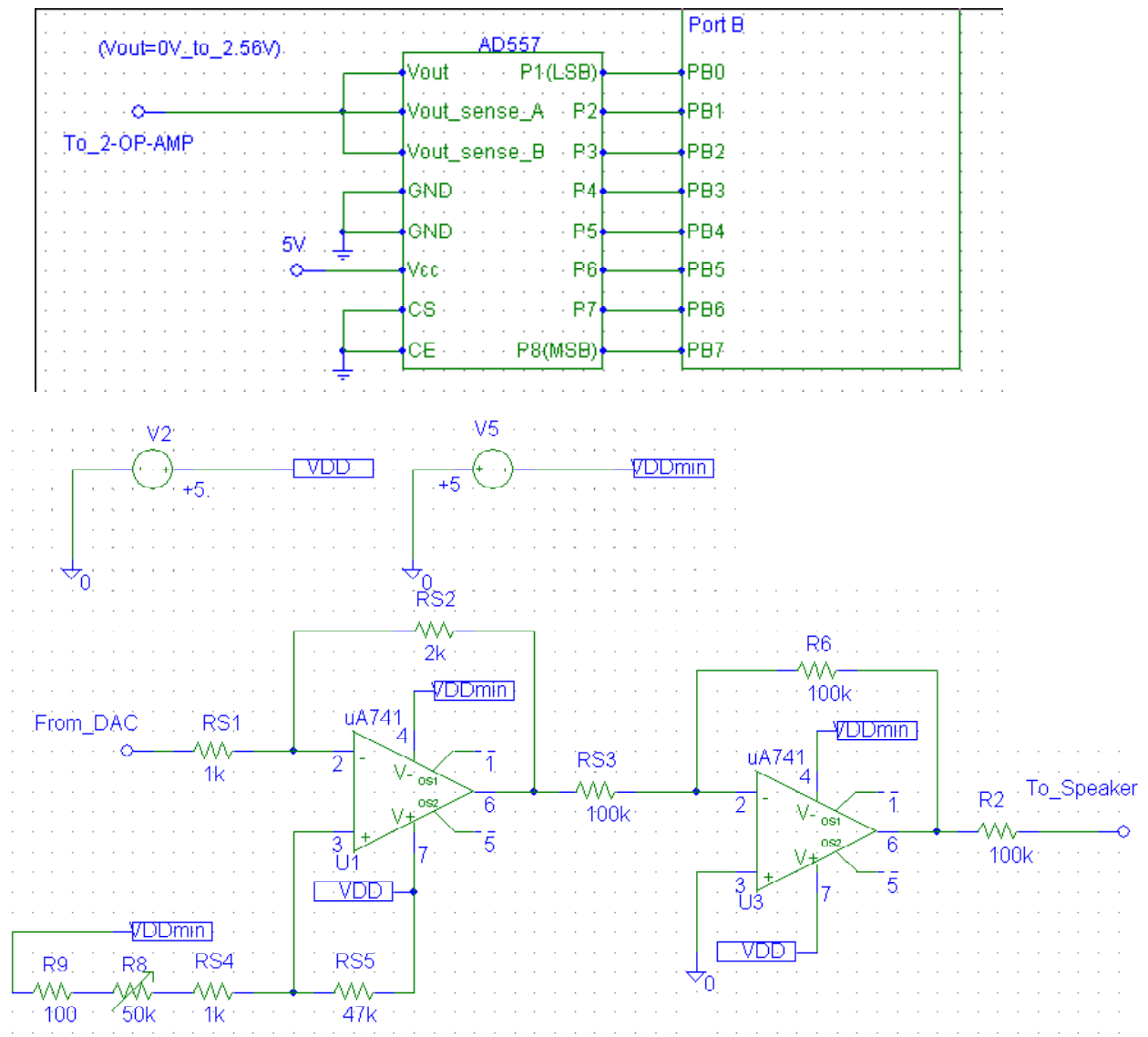

Figure 7: Output Circuit Diagram from PC Board to Speaker

As a control for the sound noise element, a CD player played a single repeating sound snippet on regular speakers. The repeating sound was to insure that each point of measurement would have little to no noticeable changes in the sound reading. Four different sound snippets of less that 2 seconds were used to measure, and each measurement was taken at the same point in the playback.

The method in which measurements were taken was to take a reading with the sound canceling circuit off, then followed by a reading with the circuit operating, then moving on to the next point. The area of the test area was a 2' x $1 \frac{1 / 2}{2}$ box that was broken into 2 "x $1 \frac{1 / 2}{2}$ grid for measuring points. The instrument used was a sound level reader, with an analog indicator, (spectrum from 0110 decibels), set to the " $\mathrm{C}$ " attenuation that represents human ears hearing response.

Measurements were either made in whole or half increments to indicate where the needle reading was on or in-between. 


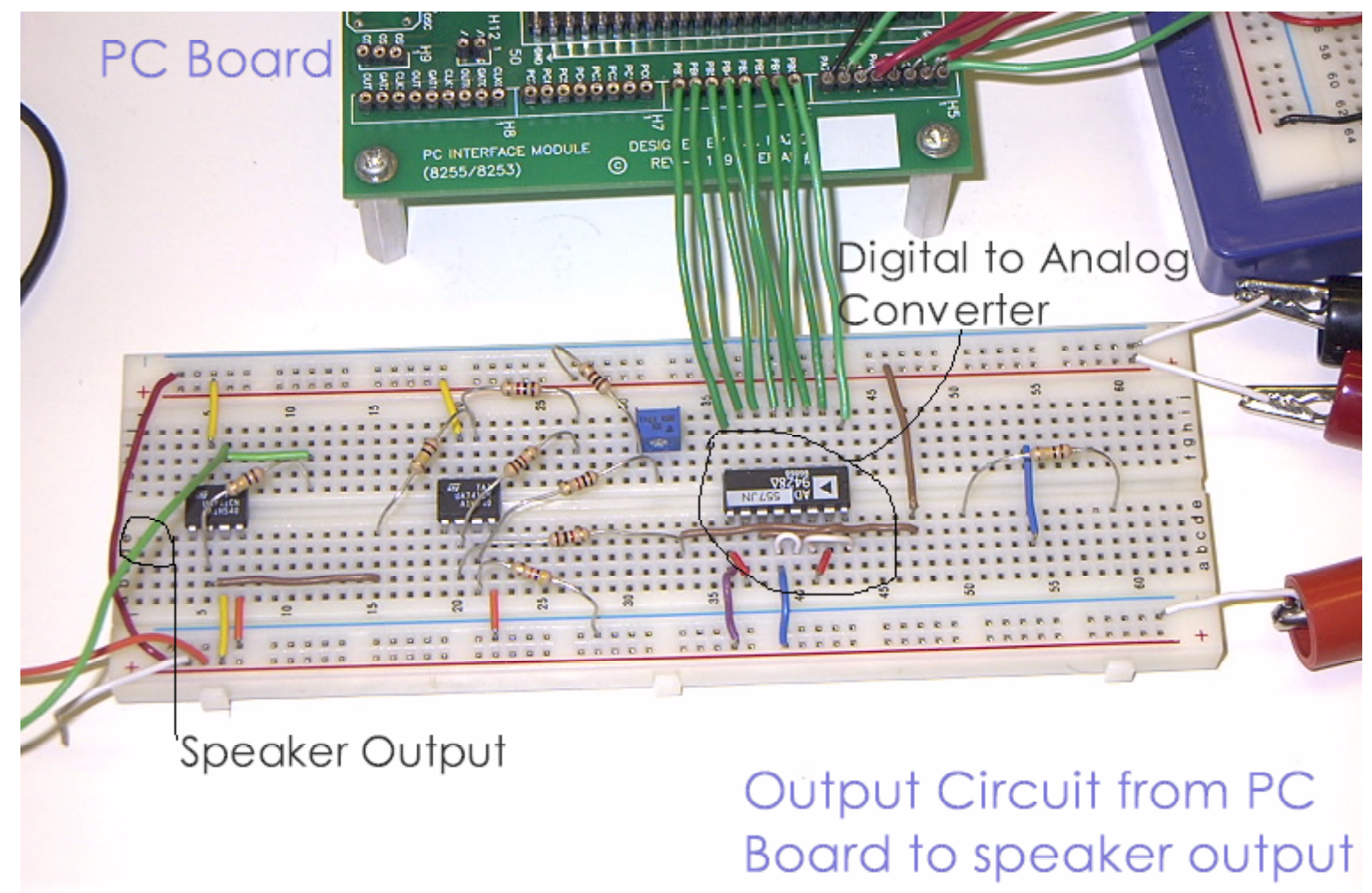

Figure 8: Actual Output Circuit From PC Board to Speaker

Experimental Data and Analysis

Data was collected in two parts; the ability of the circuit to invert sound waves from the input, and the actual ability to cancel sound in a test room. The first part used an oscilloscope to measure the input wave and output wave and visually compare them. Several types of inputs were introduced to the circuit to test if the output was correctly inverting the input. The first test introduced a signal from a sine generator directly into the input of the Analog to Digital converter, and compared that signal with actual output generated to the oscilloscope. Changing the amplitude and frequency of the sine generator was used to discover the range of accuracy of the output signal. The optimal range found by using the sine generator was an amplitude range from $0.75 \mathrm{~V}$ to $5 \mathrm{~V}$. The frequency range was from $1 \mathrm{~Hz}$ to $1.2 \mathrm{KHz}$. This is the range from which the output signal has minimal distortion due to either a weak signal or too fast a frequency for the computer. 

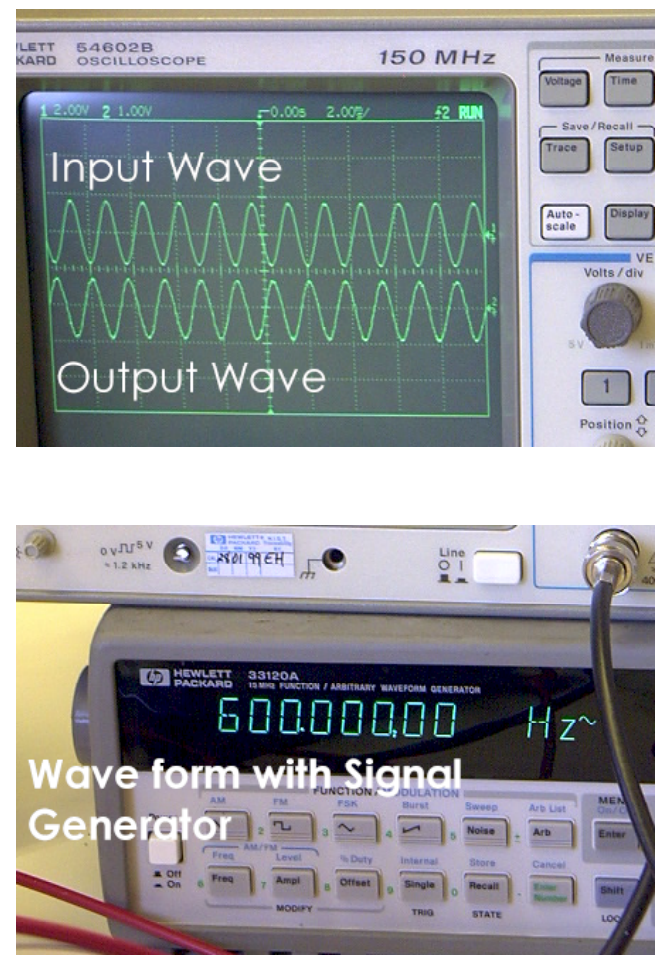

Figure 9: Signal Generator

The microphone was used for the input signal. The signal was visually checked on the oscilloscope for inversion. All testing using the microphone for input signals showed little to no distortion in the output signal. The second part was ready for testing.

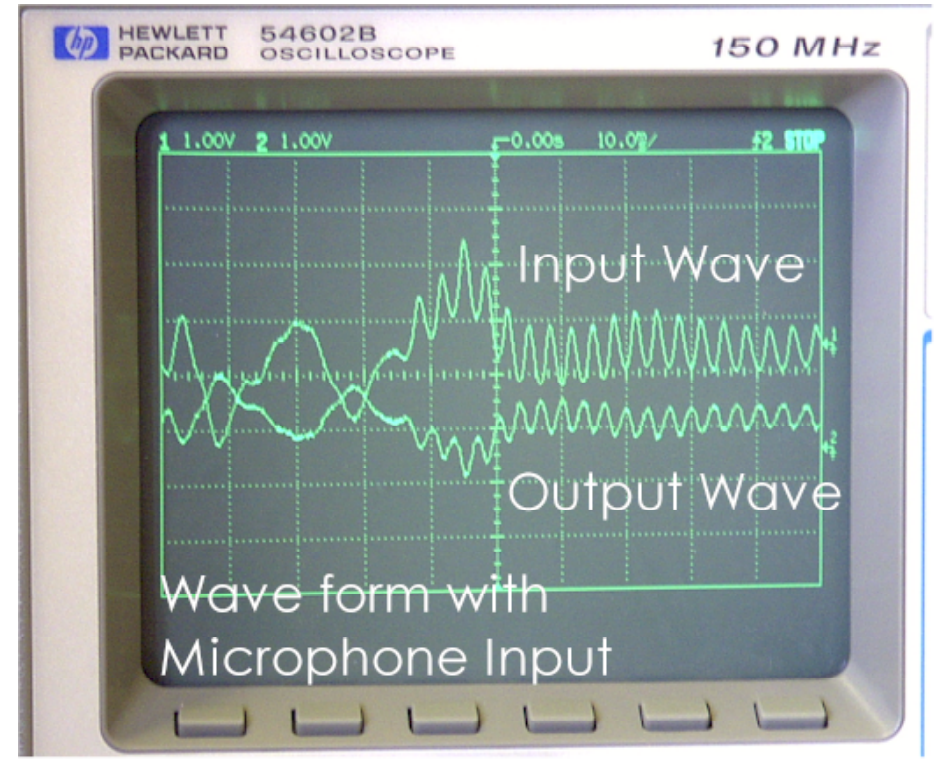

Figure 10: Sound cancellation on the oscilloscope 
The second part of the data collection comes from the decibel response of the inverting circuit. Data was collected at varying points in the boxed area using an inverted and non-inverted signal with four different sound snippets. The dead sound of the room was at a level of $57 \mathrm{db}$

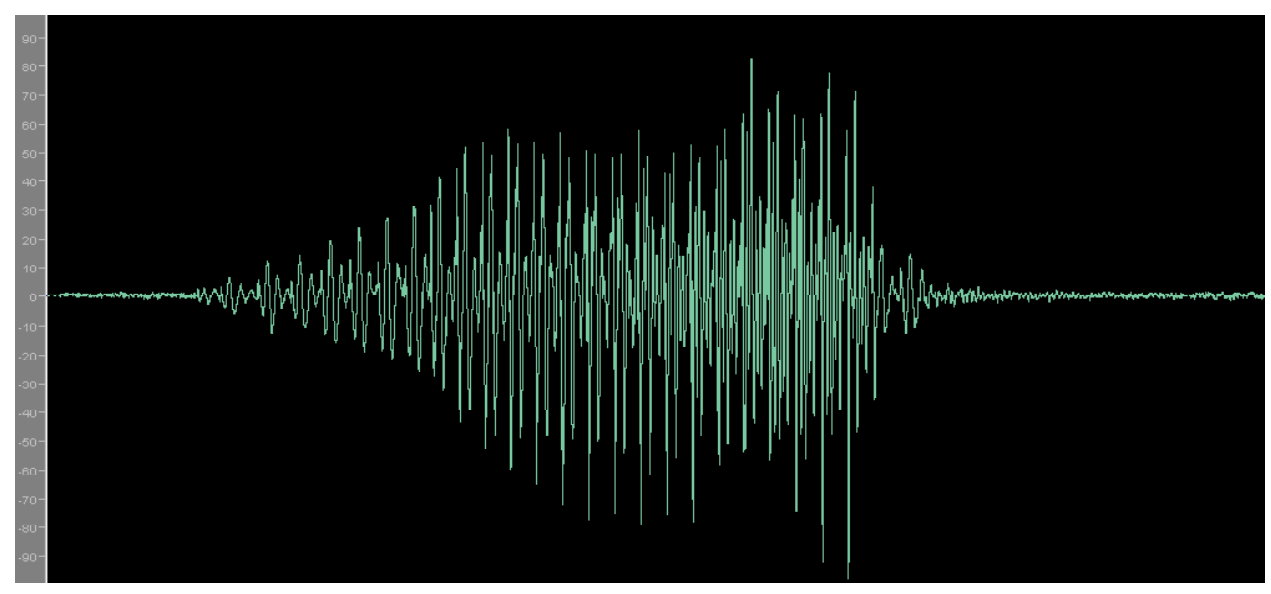

Figure 11a: Waveform Sound 1

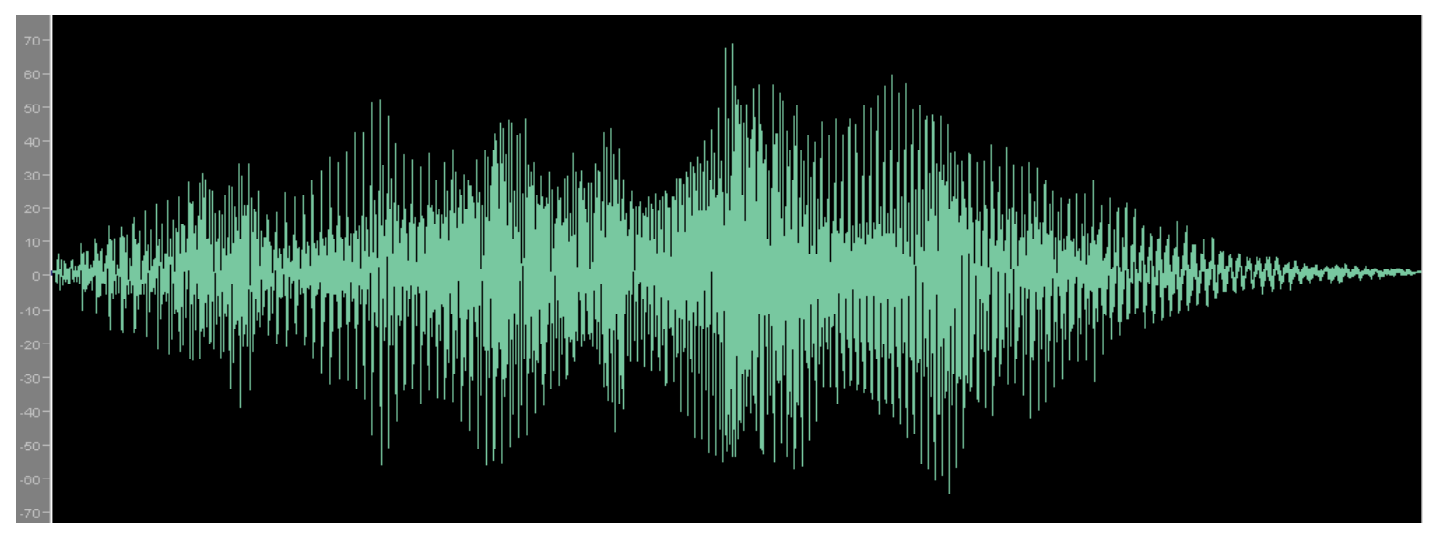

Figure 11b: Waveform Sound 2 


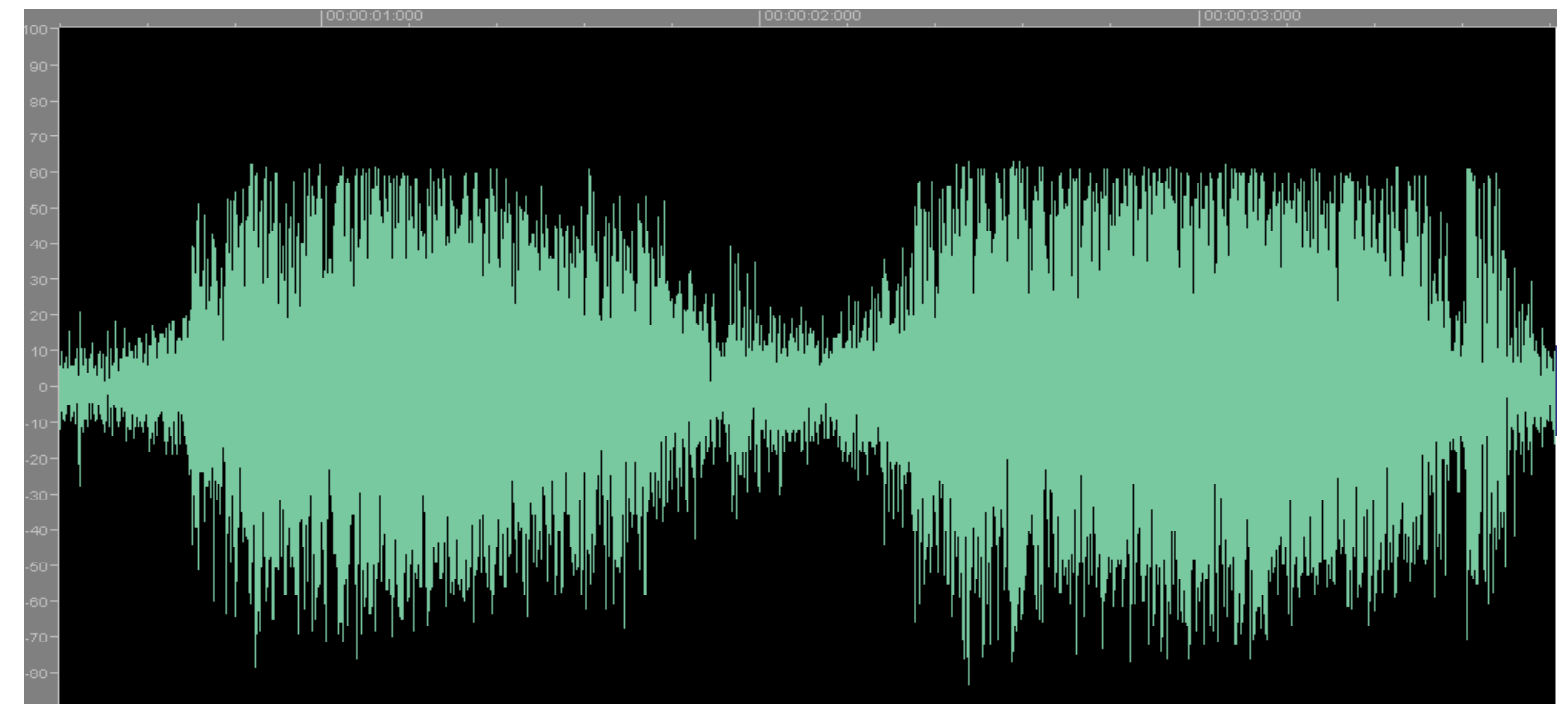

Figure 11c: Waveform Sound 3

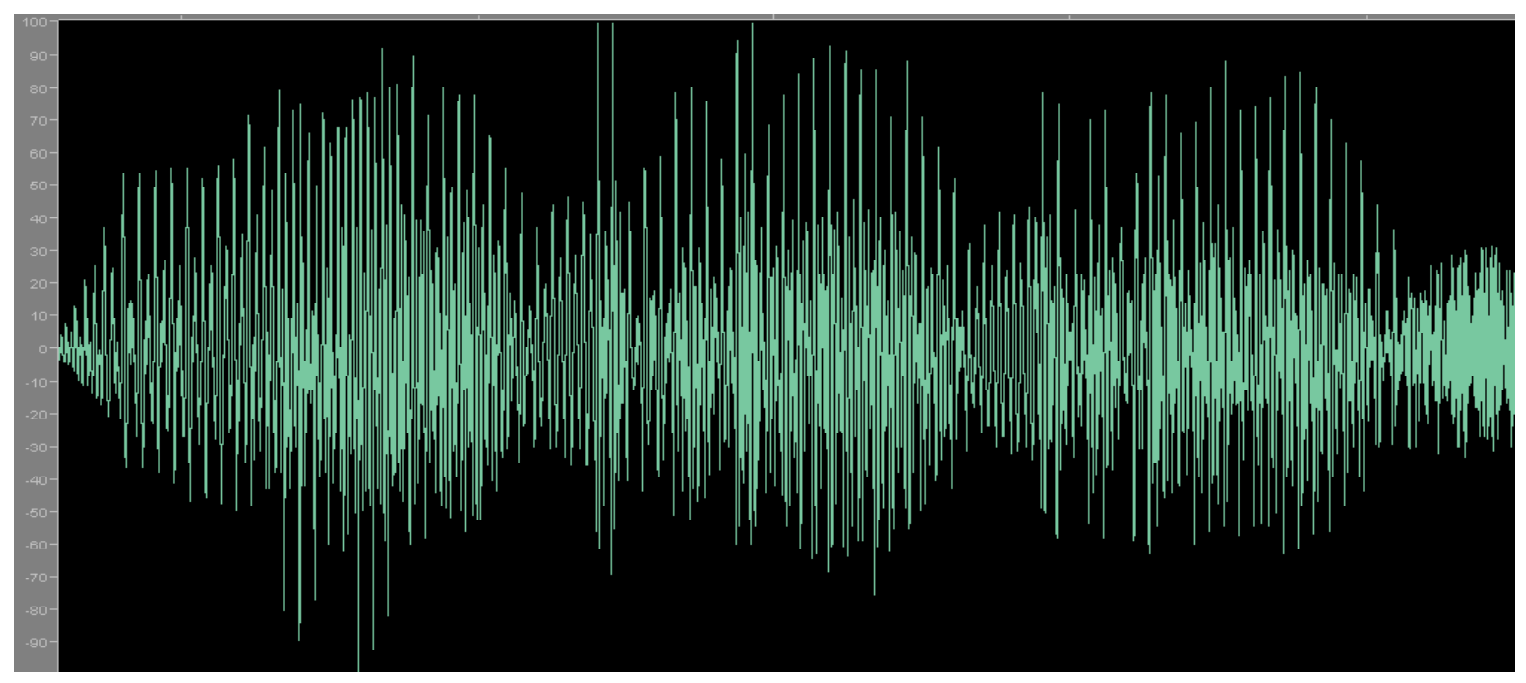

Figure 11d: Waveform Sound 4

Figure 11: Sound signatures of four snippets 
The following is the collected data:

Table 2: Sound Snippet 1:

Sound \# 1 Non-Inverted (in decibels)

\begin{tabular}{|c|c|c|c|c|c|c|c|}
\hline Inches & 2 & 4 & 6 & 8 & 10 & 12 & 14 \\
\hline 1.5 & 64.0 & 65.0 & 65.0 & 66.0 & 67.5 & 68.0 & 69.0 \\
\hline 3 & 64.0 & 64.5 & 65.0 & 65.5 & 67.0 & 68.0 & 69.0 \\
\hline 4.5 & 63.0 & 62.0 & 63.0 & 63.5 & 64.5 & 65.5 & 67.0 \\
\hline 6 & 63.0 & 62.0 & 61.5 & 61.0 & 62.0 & 63.0 & 63.5 \\
\hline 7.5 & 62.0 & 61.0 & 59.5 & 59.5 & 60.0 & 62.0 & 63.0 \\
\hline 9 & 62.0 & 61.5 & 59.5 & 59.5 & 59.0 & 61.0 & 62.0 \\
\hline 10.5 & 62.0 & 61.5 & 60.0 & 60.5 & 60.0 & 61.0 & 62.0 \\
\hline 12 & 61.0 & 61.0 & 60.5 & 60.5 & 61.0 & 62.0 & 63.0 \\
\hline 13.5 & 61.0 & 61.0 & 61.0 & 60.5 & 61.0 & 62.0 & 62.0 \\
\hline 15 & 61.0 & 62.0 & 61.0 & 60.5 & 61.0 & 61.5 & 62.0 \\
\hline 16.5 & 61.0 & 61.0 & 61.0 & 61.0 & 61.0 & 61.0 & 61.5 \\
\hline 18 & 61.0 & 61.0 & 60.0 & 60.5 & 60.5 & 61.0 & 62.0 \\
\hline 19.5 & 62.0 & 62.0 & 62.0 & 61.5 & 61.5 & 62.0 & 63.0 \\
\hline 21 & 62.0 & 63.0 & 63.0 & 64.0 & 63.0 & 64.0 & 64.0 \\
\hline
\end{tabular}

Inverted (in decibels)

\begin{tabular}{|c|c|c|c|c|c|c|c|}
\hline Inches & 2 & 4 & 6 & 8 & 10 & 12 & 14 \\
\hline 1.5 & 64.5 & 66.0 & 65.0 & 65.0 & 68.0 & 69.0 & 69.0 \\
\hline 3 & 64.0 & 65.0 & 65.0 & 65.5 & 67.0 & 68.0 & 70.0 \\
\hline 4.5 & 63.5 & 63.0 & 63.0 & 64.0 & 64.0 & 66.0 & 67.0 \\
\hline 6 & 63.0 & 63.0 & 62.0 & 62.0 & 62.0 & 63.0 & 64.0 \\
\hline 7.5 & 62.0 & 62.0 & 60.0 & 60.5 & 60.0 & 62.0 & 64.0 \\
\hline 9 & 61.5 & 61.5 & 60.0 & 60.0 & 60.0 & 61.0 & 62.0 \\
\hline 10.5 & 61.5 & 61.5 & 60.5 & 60.5 & 60.0 & 62.0 & 62.0 \\
\hline 12 & 61.0 & 61.0 & 60.0 & 60.5 & 61.0 & 62.0 & 62.0 \\
\hline 13.5 & 61.5 & 61.5 & 61.0 & 60.5 & 60.5 & 62.0 & 63.0 \\
\hline 15 & 62.0 & 62.0 & 61.5 & 61.0 & 61.0 & 61.5 & 62.0 \\
\hline 16.5 & 62.0 & 61.5 & 61.5 & 62.0 & 61.0 & 61.5 & 62.0 \\
\hline 18 & 62.0 & 62.0 & 60.5 & 61.0 & 60.5 & 61.0 & 62.5 \\
\hline 19.5 & 62.0 & 62.0 & 62.5 & 62.0 & 62.0 & 62.0 & 63.0 \\
\hline 21 & 62.0 & 64.0 & 63.0 & 64.0 & 63.0 & 64.0 & 64.0 \\
\hline
\end{tabular}
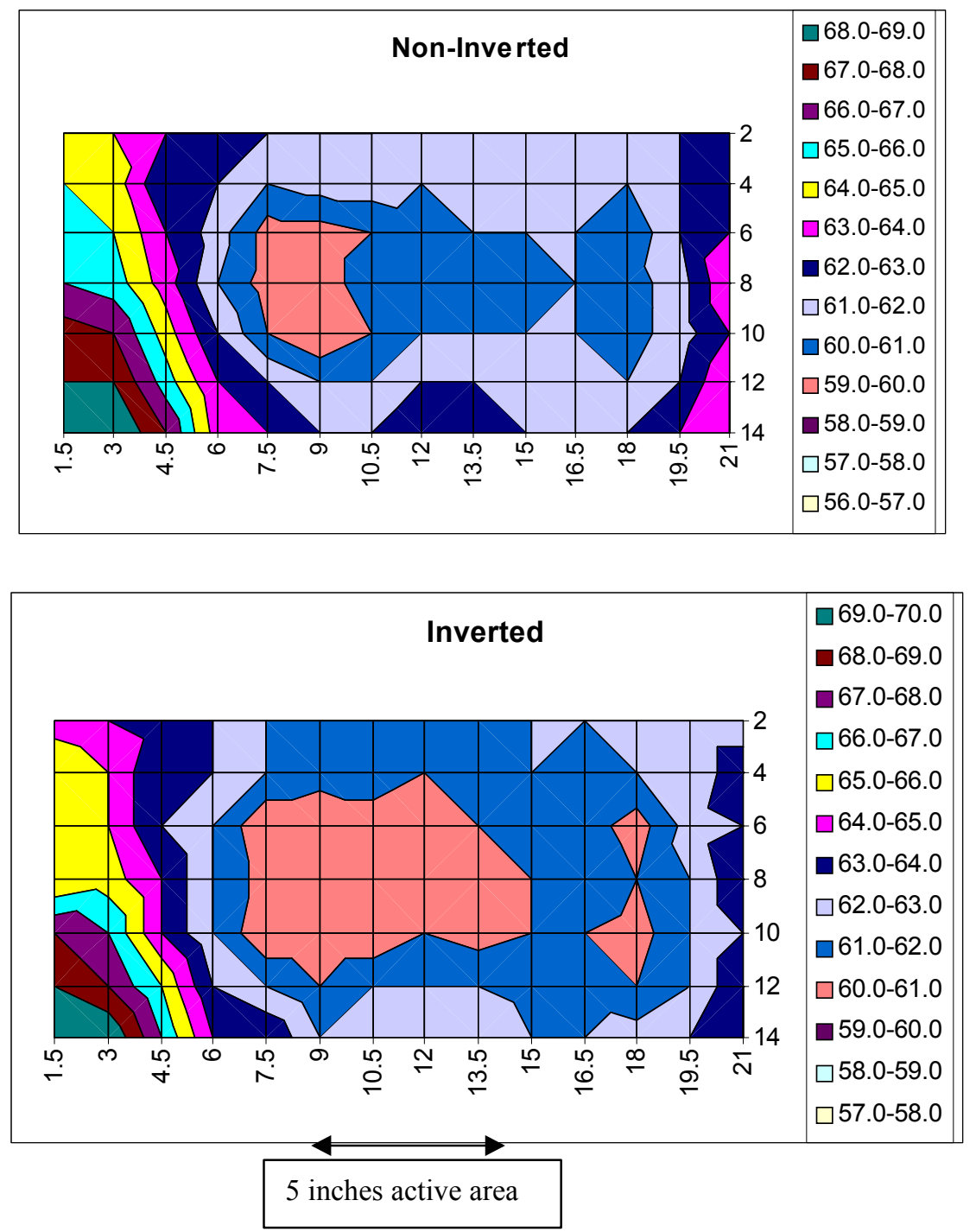

Proceedings of the 2003 American Society for Engineering Education Annual Conference \& Exposition Copyright (C) 2003, American Society for Engineering Education 
Table 3: Sound Snippet 4:

Sound \# $4 \quad$ Non-Inverted (in decibels)

\begin{tabular}{|c|c|c|c|c|c|c|c|}
\hline Inches & 2 & 4 & 6 & 8 & 10 & 12 & 14 \\
\hline 1.5 & 65.0 & 65.0 & 65.0 & 65.0 & 65.0 & 65.0 & 65.0 \\
\hline 3 & 64.0 & 65.0 & 65.0 & 64.5 & 64.0 & 64.5 & 64.0 \\
\hline 4.5 & 63.5 & 64.0 & 64.0 & 64.0 & 64.0 & 64.0 & 63.5 \\
\hline 6 & 62.5 & 63.0 & 63.0 & 63.0 & 63.0 & 63.0 & 62.5 \\
\hline 7.5 & 63.0 & 63.0 & 63.0 & 62.5 & 63.0 & 63.0 & 63.0 \\
\hline 9 & 61.5 & 62.0 & 61.5 & 61.5 & 62.0 & 62.0 & 61.5 \\
\hline 10.5 & 61.0 & 60.5 & 61.0 & 61.0 & 61.5 & 62.0 & 61.5 \\
\hline 12 & 59.5 & 60.0 & 60.0 & 59.5 & 60.0 & 61.5 & 61.0 \\
\hline 13.5 & 60.0 & 60.0 & 60.0 & 60.5 & 60.5 & 60.0 & 60.5 \\
\hline 15 & 61.0 & 61.5 & 61.0 & 61.0 & 61.0 & 61.0 & 61.0 \\
\hline 16.5 & 61.0 & 61.5 & 62.0 & 62.0 & 61.5 & 62.0 & 62.0 \\
\hline 18 & 62.5 & 62.5 & 63.5 & 63.0 & 63.0 & 63.5 & 63.0 \\
\hline 19.5 & 63.0 & 62.5 & 63.0 & 63.0 & 63.0 & 63.0 & 63.5 \\
\hline 21 & 63.0 & 63.5 & 63.0 & 64.0 & 64.0 & 63.0 & 64.5 \\
\hline
\end{tabular}

Inverted (in decibels)

\begin{tabular}{|c|c|c|c|c|c|c|c|}
\hline Inches & 2 & 4 & 6 & 8 & 10 & 12 & 14 \\
\hline 1.5 & 65.0 & 65.0 & 66.0 & 65.0 & 65.0 & 65.0 & 65.5 \\
\hline 3 & 63.5 & 64.0 & 64.5 & 64.0 & 64.0 & 64.0 & 64.0 \\
\hline 4.5 & 63.5 & 63.5 & 64.0 & 64.0 & 63.5 & 63.5 & 63.5 \\
\hline 6 & 62.0 & 62.0 & 63.0 & 63.0 & 63.0 & 62.0 & 63.0 \\
\hline 7.5 & 62.5 & 62.5 & 63.0 & 63.0 & 63.0 & 63.0 & 63.0 \\
\hline 9 & 61.0 & 62.0 & 61.0 & 61.5 & 61.5 & 62.0 & 61.5 \\
\hline 10.5 & 60.5 & 60.5 & 60.5 & 61.0 & 61.0 & 61.0 & 61.5 \\
\hline 12 & 60.0 & 61.0 & 60.5 & 61.0 & 60.5 & 61.0 & 61.0 \\
\hline 13.5 & 60.5 & 60.5 & 60.5 & 61.0 & 60.5 & 60.5 & 60.5 \\
\hline 15 & 61.0 & 62.0 & 61.0 & 61.0 & 61.0 & 61.0 & 61.0 \\
\hline 16.5 & 61.0 & 62.0 & 62.0 & 62.0 & 61.5 & 62.0 & 62.0 \\
\hline 18 & 63.0 & 63.0 & 63.0 & 62.5 & 62.5 & 63.0 & 62.5 \\
\hline 19.5 & 63.0 & 63.0 & 63.0 & 63.0 & 63.0 & 63.0 & 64.0 \\
\hline 21 & 63.0 & 63.0 & 63.0 & 63.5 & 64.0 & 64.0 & 65.0 \\
\hline
\end{tabular}
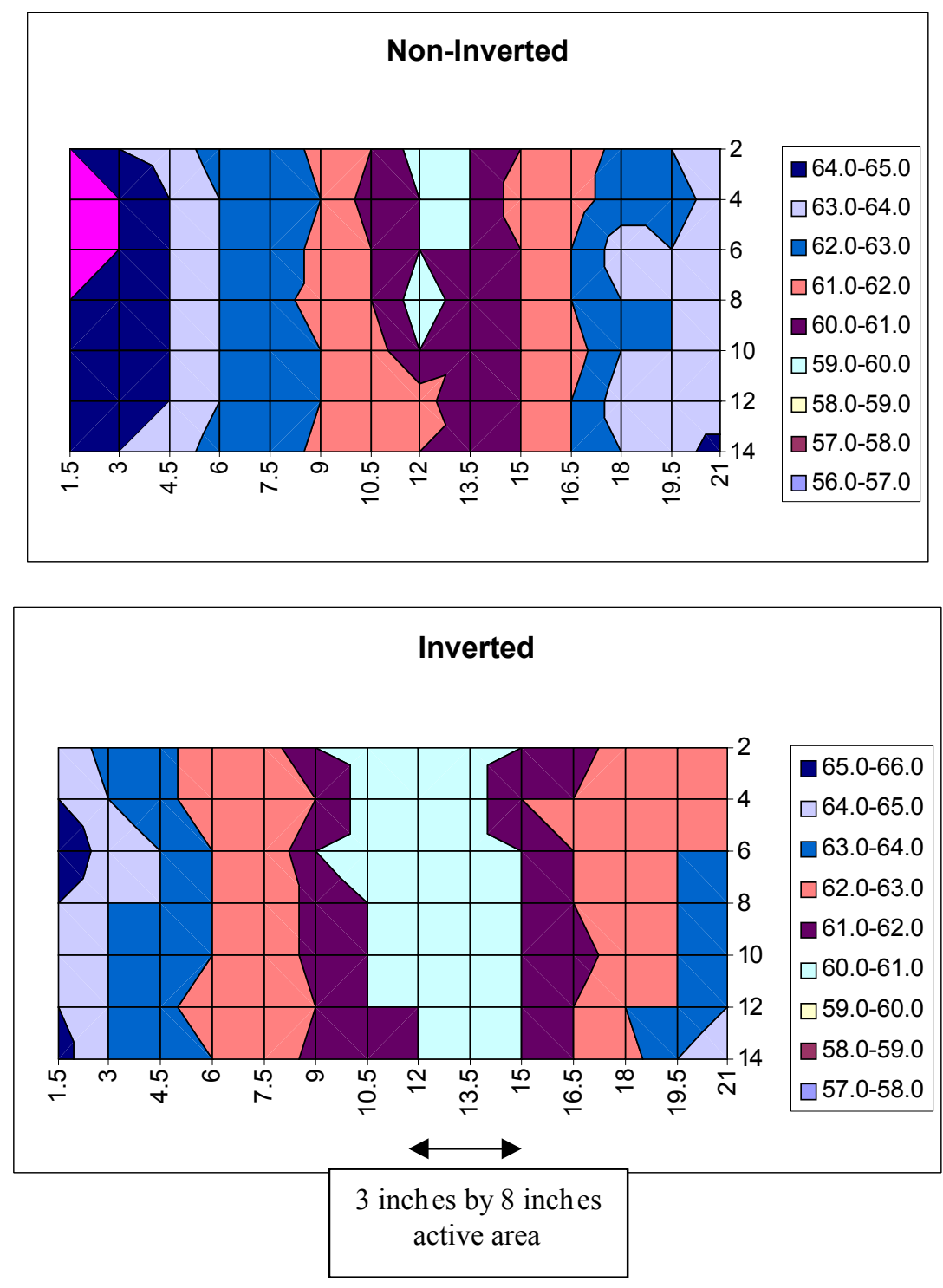

Proceedings of the 2003 American Society for Engineering Education Annual Conference \& Exposition Copyright (C) 2003, American Society for Engineering Education 
Educational Relevance of the Sound Cancellation Project

The educational relevance of this project relates to two required classes in Electrical Engineering Technology at the University of North Texas. Introduction to Microprocessors (ELET 2750) emphasizes the fundamentals of microprocessor hardware and assembly language interactions. The class uses the microprocessor to control external systems and devices. The project benefited from this class in the circuit design and testing of the hardware interface into the board microprocessor. Digital Systems (ELET 3750), utilizes microcomputers in control and instrumentation systems, including real time interfacing. Additional topics included are data communications, multiplexing, digitizing and sampling techniques. This class provided the project with the microprocessor equipment, programming techniques, and examples relative to the concept of the project. With these tools the project successfully demonstrated a measurable sound cancellation. In addition, the project concept provides a base for understanding another required course. Digital Signal Processing (ELET 3760) discusses such related topics as analog to digital conversion and back, signal sampling, digital audio processing, and DSP microprocessors.

\section{Conclusion}

The anti-noise signal will cancel itself along with the noise. A computer runs an adaptive noise control algorithm in assembly code. The program takes input and determines the corresponding value for a cancellation sound output of analog to digital and digital to analog conversions with sine wave and sound 8-bit I/O.

This system successfully receives a sound input, performs a mathematical inversion on the sound and outputs an inverted sound. The microphone circuit signal was converted through the ADC to the computer. The computer converted the signal to the inverse and output the inverted signal with little distortion when the input signal ranges was between $1 \mathrm{~Hz}$ to $600 \mathrm{~Hz}$. At frequencies over $600 \mathrm{~Hz}$ to $1.2 \mathrm{kHz}$ the distortion was noticeable.

Future Work

Even though the system successfully inverted sound input, cancellation of sound generated in the test area needs further improvement The data obtained show small change from the non-inverted to inverted decibel level. The test area will be calibrated to previous experimental setup to allow the inverted wave to match the point at which it is inverted ${ }^{12}$. Furthermore, the experimental box size will be increased by 10 .

References

1. Mazidi, Muhammad Ali; The 80x86 IBM PC and Compatiable Computers (Vol. 1 \& 2), Assembly Language, Design, and Interfacing. Prentice Hall: Upper Saddle Rive, New Jersey. 1995

2. ADC0801/ADC0802/ADC0803/ADC0804/ADC0805 8-Bit $\mu$ P Compatible A/D Converters; DataSheet; National Semiconductor; November 1999.

3. AD557 Datasheet. Analog Devices 
4. LM741 Operational Amplifier Datasheet. National Semiconductor.

5. http://www.asu.edu/clas/shs/sinex/shs311/1-sound/sound.htm

6. http://www.babcox.com/editorial/cm/cm90046.htm

7. http://virtual.parkland.cc.il.us/phy122/Fall2002Week1.doc

8. http://www.glenbrook.k12.il.us/gbssci/phys/Class/sound/u1112b.html

9. http://www.erols.com/ruckman/ancfaq.htm

10. http://arts.ucsc.edu/ems/music/tech_background/TE-06/teces_06.html

11. http://www.cavtocci.com/techtopics.htm

12. Roman Stemprok, Tan Pin-Hui, "Voice Cancellation," 2002 ASEE Annual Conference \& Exposition Proceedings, Montreal, Canada, June 18, 2002.

\section{Roman Stemprok}

Assistant Professor of Electronics Engineering Technology at the University of North Texas. Registered PE in Texas. Actively involved in research for industry with more than ten years of industrial research experience, over six years of teaching experience, ten-plus research publications. Member of TAP5-TxDOT, IESNA and CIE. M.E. in Electrical Engineering, McGill University, Montreal (1989), Ph.D. from the Department of Electrical Engineering, Texas Tech University, Lubbock (1995).

\section{SEAN DALY}

Bachelor of Science Degree in Engineering Technology from the University of North Texas expected in 2004. Part time electronic technician at UNT. 